\title{
Recent HERMES results on transverse-momentum dependent phenomena from scattering off unpolarised targets.
}

\author{
Gevorg Karyan ${ }^{1, a}$ \\ ${ }^{1}$ A. I. Alikhanyan National Science Laboratory, 375036 Yerevan, Armenia
}

\begin{abstract}
HERMES experiment at DESY in Hamburg collected a wealth of semi-inclusive deep-inelastic scattering data using the $27.6 \mathrm{GeV}$ lepton beam and pure gaseous, unpolarised hydrogen and deuterium targets. These data can be used in studies of the transverse-momentum dependent effects and can provide a check of existing models for transverse-momentum dependent parton distribution and fragmentation functions.
\end{abstract}

\section{Introduction}

Transverse momentum dependent parton distribution and fragmentation functions, usually named as TMDs, give a multidimensional description of partonic structure in momentum space. Although the studies of TMDs are mostly performed in polarized semi-inclusive deepinelastic(SIDIS) scattering processes, unpolarised SIDIS data can play an important role in studies of parton transverse-momentum dependent effects. Particularly, transverse momentum distribution of hadron multiplicities and a hadron azimuthal modulations from unpolarised targets are the key observables used in such studies.

\section{Data Selection}

The data were collected with the HERMES spectrometer using $27.6 \mathrm{GeV}$ electron or positron beams stored in HERA at DESY[1]. To select deep-inelastic scattering regime the following requirements were used for a scattered lepton : $Q^{2}>1 \mathrm{GeV}^{2}, W^{2}>10 \mathrm{GeV}^{2}$ and 0.1 $<y=v / E<0.85$, where $Q^{2}$ is the negative squared four-momentum transfer, $W^{2}$ is the invariant mass square of virtual photon-nucleon system, $v$ is the virtual photon energy and $E$ is the beam energy. The cut on the invariant mass imposed to suppress the resonance contribution into data and constrain on $y$ limits the magnitude of radiative corrections. For charged hadron identification, dual-radiator ring-imaging Čerenkov detector (RICH) was used[2], which allows to identify pions, kaons and (anti)proton. They were selected within the following kinematical conditions: $2 \mathrm{GeV}<p_{h}<15 \mathrm{GeV}$, where $p_{h}$ is a hadron momentum. Additionally, there is a cut applied on a hadron fractional energy $z$ i.e. $0.2<z<0.8$ to exclude the region at high $z$, which is dominated by exclusive processes and to suppress the target fragmentation region at low $z$.

\footnotetext{
a(for the HERMES Collaboration)e-mail: gevkar@mail.desy.de
}

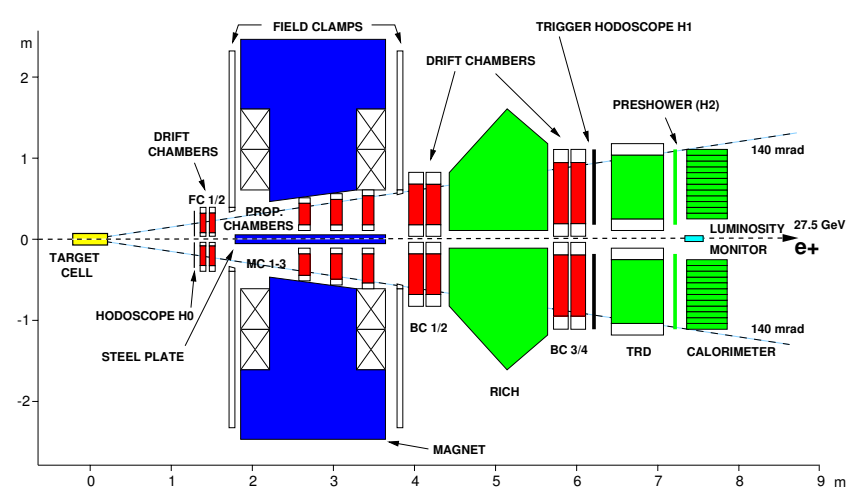

Figure 1. The HERMES spectrometer.

\subsection{Hadron multiplicities}

Hadron multiplicities i.e. hadron yields normalised to the DIS lepton yield were extracted for charged pions and koans from unpolarised hydrogen and deuterium targets[3].

$$
M^{h}\left(x_{B}, Q^{2}, z, P_{h \perp}\right)=\frac{1}{\frac{d^{2} N_{D I S}\left(x_{B}, Q^{2}\right)}{d x_{B} d Q^{2}}} \cdot \int_{0}^{2 \pi} \frac{d^{5} N^{h}\left(x_{B}, Q^{2}, z, P_{h \perp}, \phi_{h}\right)}{d x_{B} d Q^{2} d z d P_{h \perp} d \phi_{h}} d \phi_{h}
$$

The multiplicities at Born-level, i.e. no limitations in geometric acceptance and corrected for radiative and detector resolution effects, are extracted from measured multiplicities binned in 3 dimensions: $\left(x_{B}, z, P_{h \perp}\right)$. Due to the strong correlation between the $x_{B}$ and the $Q^{2}$ in the HERMES kinematical acceptance, simultaneous data binning in these two variables would not have an impact on the extraction. A $\phi_{h}$ binning has been omitted because of limited statistical precision. However, a possible impact of the $\phi_{h}$ dependence of the unpolarised semi-inclusive cross section and its acceptance have been accounted for in the systematic uncertainties. The dependencies on transverse mo- 


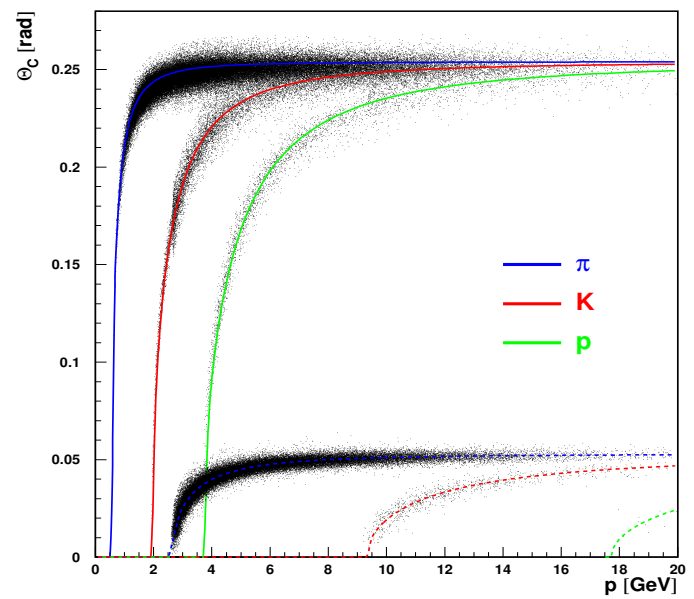

Figure 2. Momentum dependence of the Čerenkov angle for different hadron types and radiators. The upper band corresponds to the aerogel and the lower band to the $\mathrm{C}_{4} \mathrm{~F}_{10}$ gas respectively.

mentum of charged pion multiplicities are shown in Fig. 3. The features of the $P_{h \perp}$ distributions result from the com-

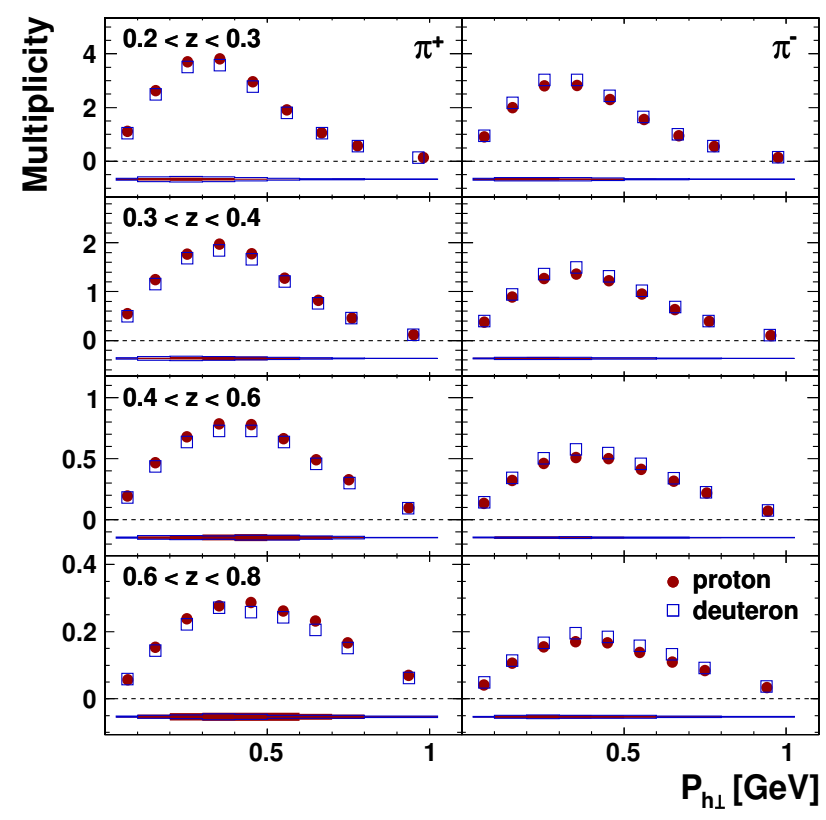

Figure 3. Multiplicities of charged pions for the proton (full circles) and the deuteron (empty squares) targets as a function of $P_{h \perp}$ in four $z$ bins. Positive charge is on the left and negative charge is on the right of the panel.

bined effects of the initial transverse motion of the struck quark in SIDIS, of its emission of soft gluon radiation, and of the component of transverse momentum which is generated by the fragmentation process. The transverse momentum depndence of charged kaon multiplicities is shown in Fig. 4. For negatively charged kaons the $P_{h \perp}$ distribution is much wider than it is for positively charged kaons which might be caused by a more extended fragmentation for $K^{-}$ which is pure sea object. Both pion and kaon multiplicities peak at increasing $P_{h \perp}$ as $z$ is increased.

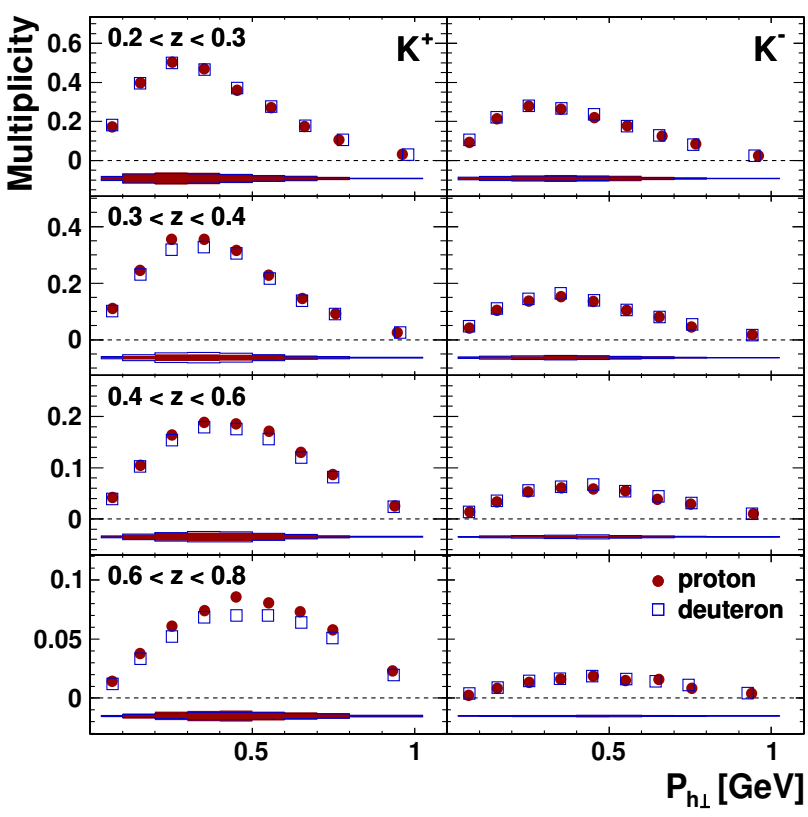

Figure 4. Multiplicities of charged kaons for the proton (full circles) and the deuteron (empty squares) targets as a function of $P_{h \perp}$ in four $z$ bins. Positive charge is on the left and negative charge is on the right of the panel.

\subsection{Azimuthal distributions of charged pions and kaons}

When one writes down unpolarised SIDIS cross section then two additional structure functions appear, one at leading twist as a $\cos \left(2 \phi_{h}\right)$ modulation and the other at subleading twist as a $\cos \left(\phi_{h}\right)$ modulation. The former one reflects a correlation between quark transverse momentum and quark transverse spin and is a convolution of the BoerMulders distribution and the Collins fragmentation func-

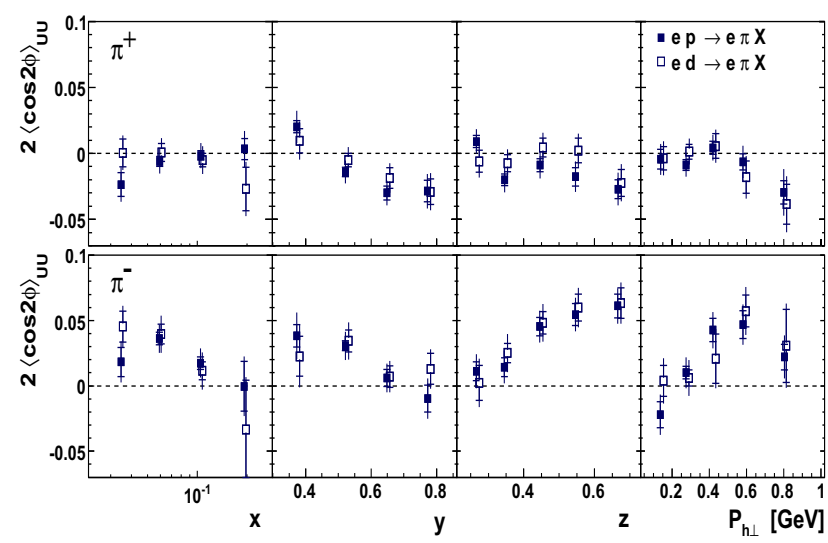

Figure 5. $\cos \left(2 \phi_{h}\right)$ moments for charged pions versus $x_{B}, y, z$ and $P_{h \perp}$ from proton target(filled symbols) and from deuteron target(empty symbols).

tion. The latter one is pure kinematic effect caused by quark intrinsic transverse momentum and contains terms 
related to the Cahn effect. The $\cos \left(\phi_{h}\right)$ and $\cos \left(2 \phi_{h}\right)$ moments were extracted from data collected on hydrogen and deuterium targets[4], using a fully differential unfolding procedure in $x_{B}, y, z$ and $P_{h \perp}$ in order to correct for detector geometrical acceptance, smearing and higher-order QED effects. In Fig. 5, $\cos \left(2 \phi_{h}\right)$ modulations are presented for charged pions. They are found to be slightly negative for positevely charged pions while for negatively charged pions the moments are positive. The $\cos \left(2 \phi_{h}\right)$ moments extracted from hydrogen and deuterium targets are found to be compartable.

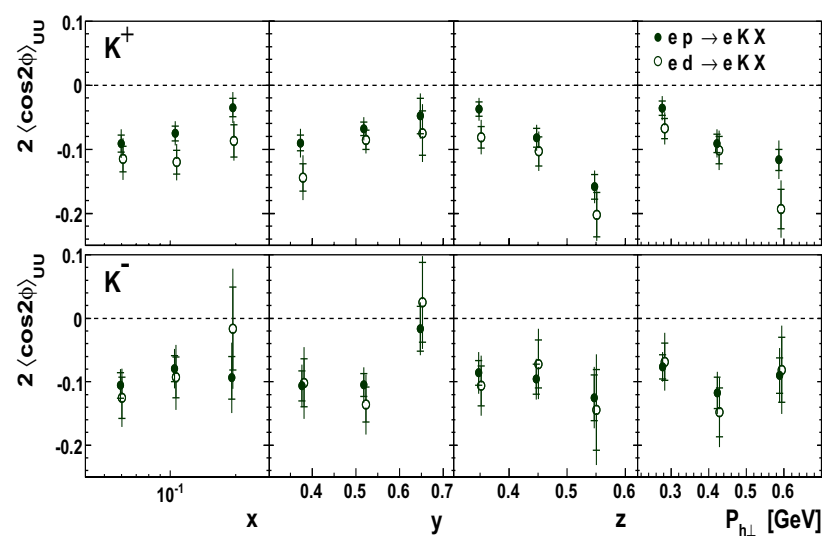

Figure 6. $\cos \left(2 \phi_{h}\right)$ moments for charged kaons versus $x_{B}, y, z$ and $P_{h \perp}$ from proton target(filled symbols) and from deuteron target(empty symbols).

Charged kaon $\cos \left(2 \phi_{h}\right)$ moments are shown in Fig. 6. They are found to be negative for both charges. The $K^{-}$ amplitude is similar to $K^{+}$amplitude and both are sig- nificantly larger than the pion amplitudes. In Fig. 7 the $\cos \left(\phi_{h}\right)$ modulations for charged pions are shown. For positively charged pions the $\cos \left(\phi_{h}\right)$ moments are negative. It is increasing with $z$ and $P_{h \perp}$. For negatively charged pions the magnitude of signal is significantly smaller in comparison with positively charged pions.

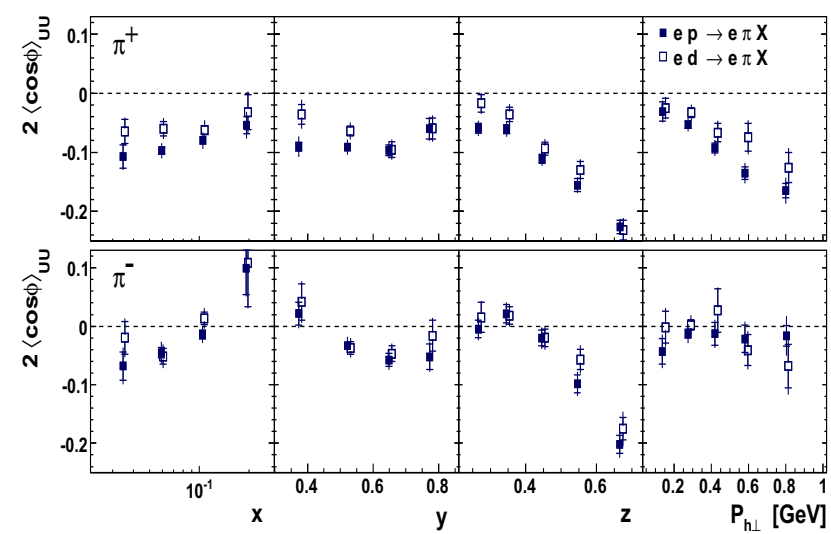

Figure 7. $\cos \left(\phi_{h}\right)$ moments for charged pions versus $x_{B}, y, z$ and $P_{h \perp}$ from proton target(filled symbols) and from deuteron target(empty symbols).

\section{References}

[1] K. Akertaff et al., HERMES coll., Nucl. Instrum. Methods A417 (1998) 239.

[2] N. Akopov et al. NIM A479 (2002) 511.

[3] A. Airapetian et al. Phys. Rev D87, (2013) 074029.

[4] A. Airapetian et al. Phys. Rev D87, (2013) 012010. 